Review

\title{
The Role of the Microbial Biofilm in the Pathogenesis of Periodontal Disease: An Update
}

\author{
${ }^{1}$ Andreas L. Ioannou, ${ }^{2}$ Konstantinos Siormpas, ${ }^{3}$ Miltiades E. Mitsias and ${ }^{4}$ Georgios A. Kotsakis \\ ${ }^{1,4}$ Advanced Education Program in Periodontology, University of Minnesota, USA \\ ${ }^{2.3}$ Private Practice, Greece
}

Article history

Received: 06-10-2014

Revised: 12-01-2015

Accepted: 26-02-2015

Corresponding Author:

Ioannou, A.L.

Advanced Education Program in Periodontology,

University of Minnesota,

USA

Email: ioann005@umn.edu

\begin{abstract}
Microbial biofilm is generally considered to be an etiologic factor for the diseases of the periodontium and has been and continues to be the main focus of avid periodontal researchers. Based on our current understanding of the role of the biofilm in the pathogenesis of periodontal disease, each different species seems to have a specific role to fulfill in this community and this chain of inter-connecting and intimately related interactions converge to shape and stabilize a disease-provoking microbiota. The aim of this review paper is to answer the specific question: "What is our current understanding on the role of the biofilm in the pathogenesis of periodontal disease".
\end{abstract}

Keywords: Gingivitis, Microbial Biofilm, Periodontal Disease, Pathogenesis

\section{Introduction}

Microbial biofilm is generally considered to be an etiologic factor for the diseases of the periodontium and has been and continues to be the main focus of avid periodontal researchers (Socransky and Haffajee, 1994).

The direct etiologic correlation between the microbial biofilm and occurrence of gingivitis has been explicitly demonstrated in the work of Loe et al. (1965). Such a profound correlation has not been causatively proven to exist between periodontitis and microbial biofilm. On the other hand, the reciprocal association has been thoroughly reported, with many studies verifying that mechanical treatment with or without adjunctive antimicrobial therapy is effective in reducing the prevalence of the majority of sub gingival species, particularly putative periodontal pathogens in patients with good response in the treatment of periodontal disease (Colombo et al., 2012; Jünemann et al., 2012). The above evidence points out that a deleterious biofilm is a key etiologic factor for periodontal disease development, but does not suffice without the co-existence of another crucial factor; host susceptibility.

The aim of this review paper is to answer the specific question: "What is our current understanding on the role of the biofilm in the pathogenesis of periodontal disease".

Discussion of the effect of host susceptibility in the occurrence of disease is outside the scope of this study, but the interaction between the biofilm and the host's immune system will be palpated.

\section{Literature Update}

Epidemiologic data have shown that the prevalence of severe periodontitis appears to fluctuate around $15 \%$ across all age groups (Pihlstrom et al., 2005). There have been numerous approaches to the study of the microcosm that exists in the crevices of teeth with active periodontal disease as well as the characterization of its lodgers (Slots, 1977a; 1977b).

In retrospective, the theories that were initially utilized to discuss the association of biofilm with disease were looking into either the biofilm's quality (specific plaquehypothesis) or quantity (non-specific plaque hypothesis).

The "non-specific plaque hypothesis" attracted significant interest in the " $50 \mathrm{~s}$ when researchers came to the conclusion that sufficient accumulation of any microorganisms below the gingival margin could cause destructive inflammation through the local production of destructive metabolites. (Schultz-Haudt and Bruce, 1954; Macdonald et al., 1956).

A good example of the inherent flaws of the "nonspecific plaque hypothesis" is $P$. gingivalis. $P$. gingivalis has enjoyed a plenitude of references in the periodontal literature due to its ease of growing in in vitro settings. (Lewis, 2010; Jain and Darveau, 2010). In a recent in vivo study it was found that low levels of P. gingivalis were able to increase the amount of commensal microorganisms in specific pathogen free mice and cause significant periodontal bone loss (Hajishengallis et al., 2011). On the 
contrary when germ-free mice were inoculated with PG no bone loss was identified. The researchers concluded that although a very minor constituent of the total microbiota, $P$. gingivalis significantly altered the numbers and community organization of the commensal bacteria, the presence of which were essential for $P$. gingivalisinduced bone loss. Therefore it seems that the role of each pathogen is not necessarily based on quantitative characteristics as in the case of low-abundance of $P$. gingivalis in the biofilm.

In the late 70 s research evidence showing that dramatic compositional changes to the microbiota in disease occur as compared with health, shifted the interest of the periodontal community to the "specific plaque hypothesis". Advocates of the theory based on the finding of (Slots, 1977a; 1977b) and others came to believe that the increase in the number of specific bacteria is the cause of periodontal disease (Slots, 1977a; 1977b). Recent studies utilizing DNA probes to identify the breadth of the microbiome of the periodontal pocket have pointed out that Porphyromonas, Tannerella and Treponema are recognized as perio pathogens, but these organisms were also found in $95 \%$ of non-diseased individuals as part of their commensal oral microbiome. (Colombo et al., 2009; Sharma, 2010; Ishihara, 2010) those findings aid in making the "specific microflora" theory obsolete since the interactions between each pathogen and the actions of immune system mechanisms are not taken into account based on this theory.

A major breakthrough in the understanding of periodontal pathogenesis and the associated microbiota was marked by the early work of Socransky et al. (1998). findings of a series of studies using whole genomic DNA probes and checkerboard DNA-DNA hybridization, lead to characterization of periodontal microbial communities on the basis of a color-coded system reflecting cluster analysis, community ordination and associated disease severity. Socransky et al. (1998) categorized periodontal associated microorganisms in 5 major complexes depending on the degree of their association with periodontal disease. The group that was most significantly correlated with inflammatory and clinical parameters of periodontal disease was given the name "red complex".

"Red complex" bacteria were Porphyromonas gingivalis, Treponema denticola and Tannerella forsythia and were strongly associated with each other and with diseased sites. This theory found solid base not only due to the individual pathogenic capacity of each of the red complex bacteria, such as the finding that Porphyromonas gingivalis causes periodontitis in nonhuman primates upon its oral implantation, but was the first one to show a level of synergy between groups of micro-organisms that characterize a shift from health to disease. A keen interest was shown by this school of thought to the key role of gram negative anaerobes in periodontally diseased sites. The historical significance of this theory lies in the introduction of the notion that an interrelated complex of species is accountable for periodontal inflammation rather than single species. The potential implication was that affecting one of these species may influence the colonization of the other species in this group, thus reducing the pathogenic potential of the microflora. The Forsyth research time recognized the limitations of their work simultaneously with its publication and wide acceptance by the periodontal world. In the 1998 publication by Socransky et al. (1998) it is noted that their investigation represents only an initial attempt at evaluating inter-relationships among subgingival species (Socransky et al., 1998). Indeed the relationships among the lodgers of the subgingival microflora was heavily investigated in the dawn of the 21 st century and it took more than a decade following the establishment of the "red complex" theory until Hajishengallis group developed the "keystone pathogen" concept which evolved to the polymicrobial synergy and dysbiosis theory (Hajishengallis et al., 2011). The foundation of this concept is that low-abundance keystone species can disrupt tissue homeostasis through quantitative and qualitative changes to the commensal microbiota. Consequently, inflammatory bone loss is mediated by the altered microbiota. The concept that certain pathogens or that a significant number of certain perio pathogens characterizes the initiation of periodontal inflammation and its destructive outcomes seems to be lending its place to emerging ideas that look into the interaction of periodontal pathogens and commensal microorganisms as a singular entity, namely the "perio-pathogenic" biofilm. Each microorganism has unique attributes and a specific role in the biofilm that determines the effect of this pathogenic stimulus to the immune system. These unique micro-organism-related attributes are associated specific molecules that provide each potential pathogens with attributes that are useful to the biofilm community and allow it to "communicate" with other pathogens in an attempt to evade the immune systems defense mechanisms. Such molecules are usually proteases, such as the gingipains associated with $P$. gingivalis pathogenicity that allows this microorganism to downregulate host response against it. Another good example of molecules that play a crucial role in synergy between different bacteria is the Chymotrypsin-Like Proteinase (CTLP) of T. Denticola. The CLTP is a surface protein that promotes colonization and virulence for $T$. Denticola and aids in its integration into biofilm 
communities by adhering to other species of oral bacteria (Cogoni et al., 2012).

These new concepts have put the "red complex dogma" to the test based on evidence that were available with the application of novel microbiological techniques. Most of the traditional periodontal microbiological studies looked into the periopathogenic microflora using either culturing techniques or "targeted-DNA" approaches. These approaches had technique-related limitations that lead to the characterization of certain species with periodontal disease while limited the discussion on species that were either impossible to culture or not targeted in analyses. Ribosomal 16S cloning and sequencing played a key role in identifying the diversity of microbial phyla associated with periodontitis, as in the study of Kumar et al., (2005). One of the very important findings of this study was that more differences were found in the bacterial profile between subjects with periodontitis and healthy subjects than between deep and shallow sites within the same subject. This suggested that chronic periodontitis is the result of a global perturbation of the oral bacterial ecology rather than a disease-site specific microbial shift.

In addition to the above, the almost exclusive "privilege" of gram-negative anaerobes to be associated with periodontal pathogenesis is largely disputed by the discovery of gram-positive anaerobic species exhibiting a significant increase in deep diseased sites relative to healthy sites. One such example is F. alocis that has been detected in greater abundance than gram-negative species in some studies (Kumar et al., 2005). A possible explanation to the belated discovery of such an abundant microbe in the periodontal pocket is the fact that it was previously not possible to cultivate it utilizing routine laboratory techniques. F. alocis may prove to have a crucial role in the pathogenesis of periodontal disease. Its pathogenic attributes include virulence properties that may enhance its ability to survive, such as favored growth in oxidative stress circumstances and persist in the periodontal pocket and may play an important role in infection-induced periodontal disease (Aruni et al., 2011).

These findings support the recognition of dental bacterial plaque as a biofilm and open new roads in the understanding of initiation of periodontal tissue destruction and the interaction of risk factors with host defenses and bacterial plaque.

\section{Conclusion}

Based on our current understanding of the role of the biofilm in the pathogenesis of periodontal disease, each different species seems to have a specific role to fulfill in this community and this chain of inter-connecting and intimately related interactions converge to shape and stabilize a disease-provoking microbiota.

It would be interesting to see focused studies aiming in understanding the interaction of the biofilm with the host-immune system and find potential new targets for therapy. Unfortunately there is a dearth of in vivo data trying to correlate specific interventions with a hopefully significant impact. Ideally, in vivo animal models should be developed based on proof-of-principle data from the current in vivo study to systematically study new treatment rationale and specific interventions.

\section{Acknowledgement}

The authors report no conflict of interest to this study.

\section{Author's Contributions}

All authors equally contributed in this study.

\section{Ethics}

This article is original and contains unpublished material. The corresponding author confirms that all of the other authors have read and approved the manuscript and no ethical issues involved.

\section{References}

Aruni, A.W., F. Roy and H.M. Fletcher, 2011. Filifactor alocis has virulence attributes that can enhance its persistence under oxidative stress conditions and mediate invasion of epithelial cells by porphyromonas gingivalis. Infect Immun., 79: 3872-3886. DOI: 10.1128/IAI.05631-11

Cogoni, V., A. Morgan-Smith, J.C. Fenno, H.F. Jenkinson and D. Dymock, 2012. Treponema denticola Chymotrypsin-Like Proteinase (CTLP) integrates spirochaetes within oral microbial communities. Microbiology, 158: 759-770. DOI: $10.1099 / \mathrm{mic} .0 .055939-0$

Colombo, A.P., S.K. Boches, S.L. Cotton, J.M. Goodson and R. Kent et al., 2009. Comparisons of subgingival microbial profiles of refractory periodontitis, severe periodontitis and periodontal health using the human oral microbe identification microarray. J. Periodontol., 80: 1421-1432. DOI: 10.1902/jop.2009.090185

Colombo, A.P.V., S. Bennet, S.L. Cotton, J.M. Goodson and R. Kent et al., 2012. Impact of periodontal therapy on the subgingival microbiota of severe periodontitis: Comparison between good responders and individuals with refractory periodontitis using the human oral microbe identification microarray. J. Periodontol., 83: 1279-1287. DOI: $10.1902 /$ jop.2012.110566

Hajishengallis, G., S. Liang, M.A. Payne, A. Hashim and R. Jotwani et al., 2011. Low-abundance biofilm species orchestrates inflammatory periodontal disease through the commensal microbiota and complement. Cell Host Microbe, 10: 497-506. DOI: 10.1016/j.chom.2011.10.006 
Ishihara, K., 2010. Virulence factors of Treponema denticola. Periodontology, 54: 117-135. DOI: 10.1111/j.1600-0757.2009.00345.x

Jain, S. and R.P. Darveau, 2010. Contribution of Porphyromonas gingivalis lipopolysaccharide to periodontitis. Periodontology, 54: 53-70. DOI: $10.1111 / j .1600-0757.2009 .00333 . x$

Jünemann, S., K. Prior, R. Szczepanowski, I. Harks and B. Ehmke et al., 2012. Bacterial community shift in treated periodontitis patients revealed by ion torrent 16S rRNA gene amplicon sequencing. PLoS ONE, 7: e41606- e41606. DOI: 10.1371/journal.pone.0041606

Kumar, P.S., A.L. Griffen, M.L. Moeschberger, E.J. Leys, 2005. Identification of candidate periodontal pathogens and beneficial species by quantitative $16 \mathrm{~S}$ clonal analysis. J. Clin. Microbiol., 43: 3944-3955. DOI: 10.1128/JCM.43.8.3944-3955.2005

Lewis, J.P., 2010. Metal uptake in host-pathogen interactions: Role of iron in Porphyromonas gingivalis interactions with host organisms. Periodontology, 52: 94-116. DOI: $10.1111 /$ j.1600-0757.2009.00329.x

Loe, H., E. Theilade and S.B. Jensen, 1965. Experimental gingivitis in man. J. Periodontol., 36: 177-187. DOI: 10.1902/jop.1965.36.3.177

Macdonald, J.B., R.M. Sutton, M.L. Knoll, E.M. Madlener and R.M. Grainger, 1956. The pathogenic components of an experimental fusospirochetal infection. J. Infect. Dis., 98: 15-20.

DOI: $10.1093 /$ infdis/98.1.15
Pihlstrom, B.L., B.S. Michalowicz and N.W. Johnson, 2005. Periodontal diseases. Lancet, 366: 1809-1820. DOI: 10.1016/S0140-6736(05)67728-8

Schultz-Haudt, S., B.G. Bibby and M.A. Bruce, 1954. Tissue-destructive products of gingival bacteria from nonspecific gingivitis. J. Dent. Res., 33: 624-631. DOI: 10.1177/00220345540330050601

Sharma, A., 2010. Virulence mechanisms of Tannerella forsythia. Periodontology, 54: 106-116. DOI: $10.1111 /$ j.1600-0757.2009.00332.x

Slots, J., 1977a. Microflora in the healthy gingival sulcus in man. Eur. J. Oral Sci., 85: 247-254. DOI: 10.1111/j.1600-0722.1977.tb00560.x

Slots, J., 1977b. The predominant cultivable microflora of advanced periodontitis. Eur. J. Oral Sci., 85: 114-121. DOI: 10.1111/j.1600-0722.1977.tb00541.x

Socransky, S.S. and A.D. Haffajee, 1994. Evidence of bacterial etiology: A historical perspective. Periodontology, 5: 7-25. DOI: 10.1111/j.1600-0757.1994.tb00016.x

Socransky, S.S., A.D. Haffajee, M.A. Cugini, C. Smith and R.L. Kent, 1998. Microbial complexes in subgingival plaque. J. Clin. Periodontol., 25: 134-144. DOI: 10.1111/j.1600-051X.1998.tb02419.x 